\title{
Technical applications of focused antenna arrays
}

\author{
Denis Vedenkin ${ }^{1, *}$ \\ ${ }^{1}$ KNRTU-KAI, RPMT Dep., 420111, Karl Marx str. 10, Kazan, Russia
}

\begin{abstract}
We analysed possibility of using focused antenna arrays at some technical applications. For example, conveyor method of material processing, wood processing for disinfection. Using principles of focusing antenna arrays can increase efficiency of modern microwave technological equipment against classical microwave processing devices.
\end{abstract}

\section{Introduction}

Currently, an increasing number of devices and systems use electromagnetic fields in their work. The quality of the formation and spatial distribution of these fields allows us to achieve the required functional indicators of microwave devices and systems. Improving characteristics of antenna devices that form electromagnetic fields, in turn, will also improve the technological parameters of promising microwave devices. One of the options for implementing new types of microwave devices may be use the principle of focused aperture. The idea of focused aperture lies in the addition of partial electromagnetic fields from elementary sources at the focal point. The principle of formation and some properties of the focused field are considered in a number of works [1-4]. The purpose of this article is to analyze possible technical applications with using focused antenna arrays.

\section{Mathematical model of focused antenna array}

A focused antenna array consists of several elementary sources. During their work they are emitting electromagnetic fields with a specially defined amplitude-phase distribution. The choice of the type of amplitude-phase distribution depends on several parameters, for example:

- distance to the focusing point;

- intensity of the focused electromagnetic field

- size of the focusing region, etc.l

Elementary sources in the general case can be located arbitrarily within the volume of a certain geometric body. One of the versions of the model of a focused antenna array is presented on Fig. 1.

* Corresponding author: denis_ved@mail.ru 


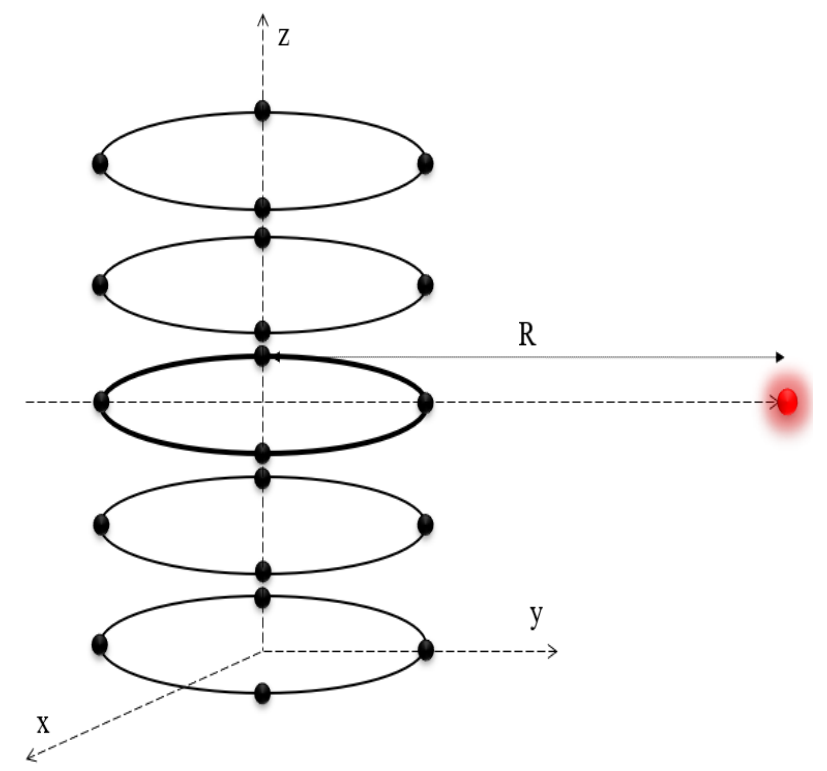

Fig. 1. Model of focused antenna array.

The main parameters of the mathematical model include:

- the number of elementary radiators;

- type of amplitude-phase distribution;

- dimensions of the antenna array;

- distance to the focus point.

\section{Conveyor processing with using a principle of focused aperture}

For typical technological problems associated with the conveyor method of processing materials and substances, the main parameters are the width, length and speed of the conveyor belt. These parameters determine the amount of raw material to be processed. Moreover, in classical microwave conveyor systems, the creation of a static amplitude distribution of the electromagnetic field is implied, and its change is associated primarily with the properties of the processed substance. Using the principle of focused aperture gives us possibility of controlling the amplitude distribution of the electromagnetic field in order to intensify the effect by changing the position of the focus point. Model of a conveyor section with four emitters of a focused antenna array and power flow distribution is shown on Fig. 2. Excitation signals of emitters are in-phase. The transverse distribution of the electromagnetic field in the thickness of the processed substance is shown on Fig. 3.

As can be seen from Figures 2, 3, the creation of a focused field is possible. We show the ability to control the focused field, for this we change the phases of the first emitter. The results are presented on fig. 4.

As can be seen from fig. 4., there was a change in the amplitude distribution of the electromagnetic field with the appearance of a pronounced minimum.

Thus, the use of the principle of focused aperture makes it possible to increase the efficiency of microwave complexes by up to $30 \%$. As an illustration (Fig. 5.), we present the results obtained by modeling the effects of the antenna array on a water-oil emulsion.

Also, some results were published in $[5,6]$. 


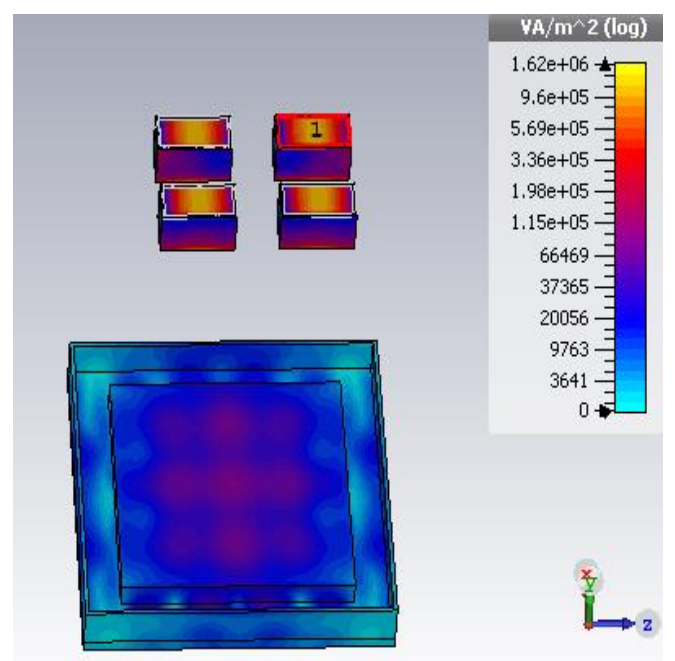

Fig. 2. The distribution of the power flow of a focused antenna array with four emitters.

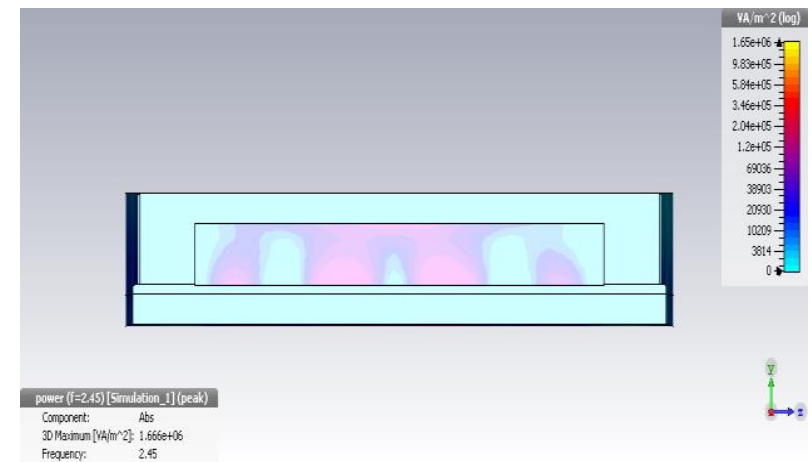

Fig. 3. The distribution of the electromagnetic field in the thickness of the processed substance.

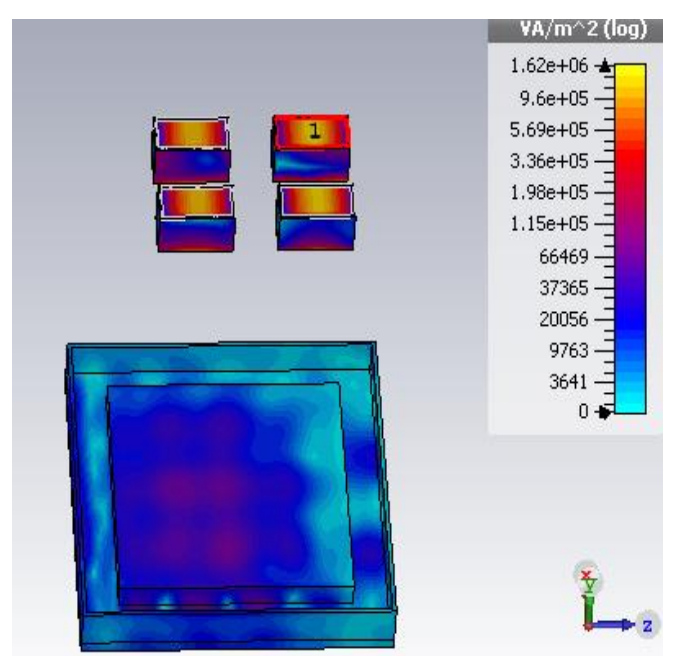

Fig. 4. Power flow distribution of a focused antenna array with non-phase excitation. 

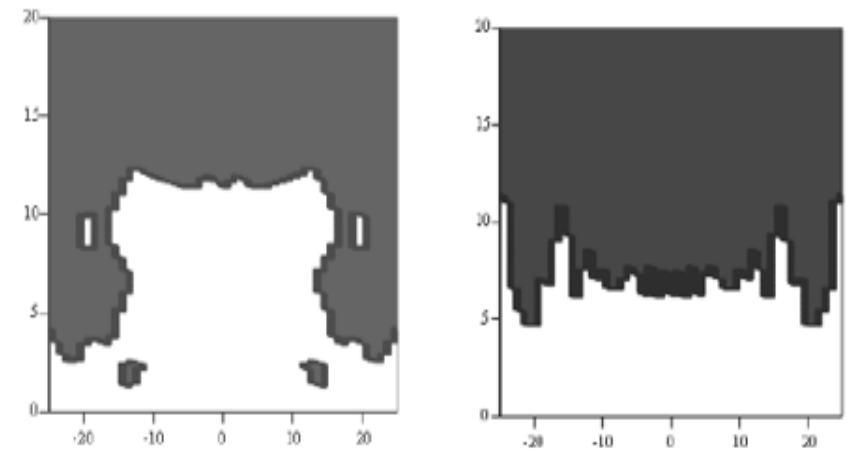

Fig. 5. The boundaries of the change in the processed volume of the oil-water emulsion: using the principle of focused aperture (left), using classical microwave heating (right).

\section{Sanitary wood processing using the principle of focused aperture}

Widespread use in the construction and architecture of wood products requires reliable protection of wood from adverse factors. At the same time, various protective compounds are used to protect the wood, which are applied to its surface and require annual renewal. However, not all of the existing protective compounds prevent the development of larvae of woodworm beetles and other insects that infect wood. One of the options for improving the operational properties of wood products and their protection against insect pests may be the use of focused antenna arrays. The advantage of this method is the possibility of implementing directed radiation focused on pests located in the thickness of the product. Research is currently underway in this area.

\section{Conclusion}

As a result of the studies, it was shown that using principle of focused aperture can increase the potential of microwave technological complexes. Their use is advisable in solving a number of existing and promising tasks. Some of them are described in works, for example, in [7].

\section{Acknowledgements}

This work was financially supported by the Ministry of Science and Higher Education of the Russian Federation as part of the basic part of State Assignment № 8.6872.2017 / БЧ.

\section{References}

1. Vedenkin D.A., Potapova O.V., Sedelnikov Y.E., 9th International Conference on Antenna Theory and Techniques, ICATT 2013 - Proceedings Antennas, focused in the near radiated field zone. Features and technical application, pp. 560-565 (2013)

2. Vedenkin D.A., Sedelnikov Y.E., Nasybullin A.R., Journal of Telecommunications and Information Technology, Antenna arrays focused on broadband signals, 2, pp. 95-102 (2016)

3. Vedenkin D.A., Nasybullin A.R., Sedelnikov Y.E.,, 11th International Conference on Antenna Theory and Techniques, ICATT 2017 - Proceedings, Random incoherent antenna arrays focused in the near field zone, pp. 159-161 (2017). 
4. Vedenkin D.A., Nasybullin A.R., Ivanov V.A., Ryabova M.I., Systems of Signal Synchronization, Generating and Processing in Telecommunications, SINKHROINFO 2017, Properties of volume non-coherent focused antenna arrays, pp. 411-413 (2017).

5. Vedenkin D.A., Samoshin R.E., Zuev O.Yu., International Conference on Antenna Theory and Techniques: Dedicated to 95 Year Jubilee of Prof. Yakov S. Shifrin, ICATT 2015 - Proceedings, Processing of oil sludge using microwave energy, pp. 399401 (2015)

6. Vedenkin D.A., Sharonov D.E., 11th International Conference on Antenna Theory and Techniques, ICATT 2017 - Proceedings, Investigation of processing of dense oily sludge using microwave energy, pp. 437-439 (2017)

7. Vedenkin D.A., Sedelnikov Y.E., Testoedov N.A., Romanov A.G., Kudymov V.I., IOP Conference Series: Materials Science and Engineering, Focused antennas for diagnostics of spacecraft antenna systems (2018) 